\title{
Harmonization of malaria rapid diagnostic tests: best practices in labelling including instructions for use
}

Jan Jacobs ${ }^{1,2^{*}}$, Barbara Barbé ${ }^{1}$ Philippe Gillet ${ }^{1}$, Michael Aidoo $^{3}$, Elisa Serra-Casas ${ }^{1}$, Jan Van Erps $^{4}$, Joelle Daviaud ${ }^{5}$, Sandra Incardona ${ }^{6}$, Jane Cunningham ${ }^{7}$ and Theodoor Visser ${ }^{8}$

\begin{abstract}
Background: Rapid diagnostic tests (RDTs) largely account for the scale-up of malaria diagnosis in endemic settings. However, diversity in labelling including the instructions for use (IFU) limits their interchangeability and userfriendliness. Uniform, easy to follow and consistent labelling, aligned with international standards and appropriate for the level of the end user's education and training, is crucial but a consolidated resource of information regarding best practices for IFU and labelling of RDT devices, packaging and accessories is not available.

Methods: The Roll Back Malaria Partnership (RBM) commissioned the compilation of international standards and regulatory documents and published literature containing specifications and/or recommendations for RDT design, packaging and labelling of in vitro diagnostics (IVD) (which includes RDTs), complemented with a questionnaire based survey of RDT manufacturers and implementers. A summary of desirable RDT labelling characteristics was compiled, which was reviewed and discussed during a RBM Stakeholder consultation meeting and subsequently amended and refined by a dedicated task force consisting of country programme implementers, experts in RDT implementation, IVD regulatory experts and manufacturers.
\end{abstract}

Results: This process led to the development of consensus documents with a list of suggested terms and abbreviations as well as specifications for labelling of box, device packaging, cassettes, buffer bottle and accessories (lancets, alcohol swabs, transfer devices, desiccants). Emphasis was placed on durability (permanent printing or water-resistant labels), legibility (font size, letter type), comprehension (use of symbols) and ease of reference (e.g. place of labelling on the box or cassette packaging allowing quick oversight). A generic IFU template was developed, comprising background information, a template for procedure and reading/interpretation, a selection of appropriate references and a symbol key of internationally recognized symbols together with suggestions about appropriate lay-out, style and readability.

Conclusions: The present document together with its additional files compiled proposes best practices in labelling and IFU for malaria RDTs. It is expected that compliance with these best practices will increase harmonization among the different malaria RDT products available on the market and improve their user-friendliness.

Keywords: Malaria RDT, Harmonization, Best practices, Labeling, Instructions for use

\footnotetext{
*Correspondence: jjacobs@itg.be

'Department of Clinical Sciences, Institute of Tropical Medicine, Antwerp,

Belgium

${ }^{2}$ Department of Microbiology and Immunology, University of Leuven,

Leuven, KU, Belgium

Full list of author information is available at the end of the article
} 


\section{Background}

\section{Global malaria burden and importance of diagnostic testing}

As the global burden of malaria is decreasing, diagnostic testing for malaria before starting treatment is crucial to avoid overtreatment with anti-malarials and delayed management of non-malaria febrile illnesses. To this end, since 2010, the World Health Organization (WHO) broadened its recommendations for parasitological confirmation of malaria diagnosis before treatment to all age groups [1,2]. Furthermore, in 2012, the “T3: Test. Treat. Track." initiative called to scale-up diagnostic testing, better target treatment and improve surveillance systems [3].

\section{Scale-up of malaria diagnosis has mainly been achieved by rolling-out of RDTs}

Case management treatment guidelines recommend diagnostic testing by use of quality light microscopy or alternatively by quality rapid diagnostic tests (RDTs) [2]. RDTs are instrument-free test devices (primarily a plastic cassette that encloses a test strip) capable of providing results within 30 minutes. They require minimal training and expertise compared to microscopy and can be performed by less-skilled health workers [4,5]. In endemic settings, diagnosis of Plasmodium falciparum infections made by wellperforming RDTs has been shown to be equal or superior to routine microscopy, and appears to be more costeffective than microscopy [6-10]. RDTs are largely accountable for the scale-up of parasitological diagnosis $[1,5,11]$.

\section{Limitations of RDTs: variety of procedures, limited user-friendliness}

Despite their robustness and apparent simplicity, RDTs have their limitations [12]. Surveys suggest there are currently at least 200 RDT products on the market $[13,14]$, which vary both in type and design of test device (shape of cassette, number of wells), accessories (specimen transfer devices, lancets) and procedure (specimen volume, numbers of buffer drops, reading time). In addition, there are many differences in labelling and terminology for the RDT box, device packaging, buffer bottles and accessories [5]. This diversity contributes to the limitations in terms of user-friendliness especially since RDTs are often performed by less-skilled heath workers. Such differences create challenges in terms of training when operators are required to switch from one RDT product to another. Problems with operating different RDT products are particularly noted during the early phases of RDT introduction at the country level [5].

\section{Harmonization and enhanced user-friendliness of RDTs}

Enhanced harmonization of malaria RDT characteristics is expected to facilitate procurement and supply management as well as to reduce training/re-training and supervision when switching from one RDT product to another or when two or more RDT products are concurrently used in a country. In addition, it is expected to improve general adherence to manufacturer's recommended procedures and reduce operational errors. Uniform, easy to follow and consistent labelling and instructions for use (IFU) are crucial to a good performance of the test and to harmonization of RDTs [15], but a comprehensive resource containing specific information about best practices in instructions for use, labelling, of RDT devices, packaging and kit accessories is not available.

In July 2012, the Diagnostics Work Stream of the Roll Back Malaria Partnership (RBM) Procurement and Supply Chain Management Working Group commissioned the Institute of Tropical Medicine (ITM, Antwerp, Belgium) to assess the level of similarities and differences between current commercially available malaria RDTs, and to identify opportunities and challenges for enhanced and rational harmonization of malaria RDT characteristics. As a guidance for the latter, regulatory documents (such as legislation, regulatory guidance and standards) and published literature relating to design, packaging and labelling of in vitro diagnostics (IVD) were compiled and assessed for their applicability and feasibility to incorporate into malaria RDTs. The process and results of these findings are described here; the results of the comparative assessment of commercially available RDTs and accessories against these findings is presented elsewhere.

\section{Methods}

Working document of best practices in labelling including instructions for use of RDTs

In a first step, regulatory guidance and guidelines for medical devices were identified and assessed for applicability for malaria RDTs. They included documents issued by the Global Health Task Force (GHTF) (now International Medical Device Regulators Forum (IMDRF)) [15-17], the International Organization of Standardization (ISO norms) [18-20], the European Commission (EC directives) [21-23], the USA Food and Drug Administration (FDA) (http://www.fda.gov/) regulations relating to labelling of IVDs [24] and a the GP42-A6 guidelines (Procedures and devices for the collection of diagnostic capillary blood specimens) from the Clinical and Laboratory Standards Institute (CLSI) [25]. In addition, information about end-user errors in RDTs and readability of instructions was retrieved from scientific literature and complemented with ITM field observations and comments from manufacturers and implementers obtained through face-to-face structured interviews (five manufacturers and 9 implementers) and an internet-based questionnaire (16 implementers). 
Based on this review, the ITM team drafted a working document compiling suggestions for consideration towards enhanced harmonization and user-friendliness, addressing issues of labelling and design and construction of RDT cassette and accessories. Interviews with manufacturers, based on these suggestions, were conducted and manufacturers rated the considerations for relevance and feasibility for incorporation into manufacturing processes. Full details of methods and sources can be retrieved on the RBM website [26].

\section{Consultation meeting with malaria control programme implementers, manufacturers and experts in regulatory affairs}

The same ITM working document was extensively discussed during a RBM Stakeholder Consultation meeting (December $3^{\text {rd }}-5^{\text {th }} 2013$, ITM) through small group and plenary review sessions. The meeting gathered 81 participants representing (i) country programme implementers (laboratory managers from the National Malaria Control Programmes (NMCP), laboratory experts involved in RDT implementation research, $n=27$ ), (ii) experts involved in global support to RDT implementation ( $n=16)$, (iii) experts in IVD regulatory matters $(n=22)$ and (iv) RDT manufacturer representatives (16 participants from nine companies), involved in research and development, quality assurance, production or business development and with sales estimated to represent over $90 \%$ of the current public market in 2010 [27].

The meeting resulted in a consolidated list of suggested specifications promoting harmonization and set out further steps and timelines towards enhanced harmonization of RDT characteristics [28]. To ensure follow-up, the meeting endorsed the RBM Procurement and Supply Chain Management Working Group to create an ad-hoc taskforce and participants were invited to express their interest to join this taskforce.

\section{MRDT harmonization task force (HarT)}

The "mRDT Harmonization Taskforce" (HarT) comprised implementers $(n=8)$, manufacturers $(n=4)$ and regulatory experts $(n=19)$; its mandate included finalization of the suggestions for interchangeability and user-friendliness of RDTs. Between February and June 2014, HarT refined the priorities for labelling and IFU and delivered consensus documents including a list of harmonized terminology and abbreviations, specifications for labelling of RDT box, buffer bottle, device packaging and accessories, as well as a generic template for IFU.

\section{Ethical clearance}

The project was submitted to and approved by the Institutional Review Board of the Institute of Tropical Medicine $(851 / 12)$.

\section{Results \\ Working document of best practice suggestions for labelling including IFU of malaria RDTs}

Figure 1 gives an overview of regulatory documents relating to labelling and IFU for IVDs. Standards and norms, guidelines and suggestions were assessed according to the following subjects: general requirements, content of the label, specific labelling of RDT box, packaging, buffer bottle, cassette and accessories, and IFU. Based on their assessment, the final working document included 66 suggestions of which more than two-thirds $(n=46,70 \%)$ were related to labelling and IFU [26]. Of these 46 suggestions, 33 (71.7\%) were sourced from regulatory documents and/or published literature, another 12 were made by implementers and the remaining one was made by a manufacturer. In interviews with manufacturers, most suggested requirements (33/46, 72\%) were scored as "highly relevant" and "feasible to align with in the short term" (i.e. within one year).

\section{Outcomes of consultation with RDT implementers, manufacturers and experts in regulatory matters}

The recommendations compiled in the working document were modified and refined during the consultation meeting. Some implementers' suggestions were not seen as critical, amongst them the recommendations (i) to use an identical lot number for box, cassette packaging and buffer vial, (ii) to display a version number of IFU on the RDT box or device packaging, (iii) to add a revision history to the IFU and (iv) to affix the date of production (except in case of national regulatory requirement). Likewise, the recommendations to display all essential information (including custom/variable information) grouped together on at least two sides of the box and to print all text and symbols in the same direction (to facilitate visual capture at inspection) were withdrawn as well as a minimum interline space of 2 for print formatting for the IFU. The consultation report was published on the RBM website [28].

Interviews with implementers confirmed that the plethora of RDT products was perceived as a major operational problem, because of variations in test procedures and because of differences in reading and interpretation of results (e.g. the characters used for the reading legend and for the labelling of the sample and buffer wells).

\section{mRDT Harmonization task force (HarT) consensus documents}

The HarT further elaborated the consultation report and developed consensus documents for RBM, presented as additional files herein. Additional file 1 lists the recommended terms and abbreviations related to malaria RDTs, Additional file 2 lists the recommended requirements for 


\section{Source of regulatory documents relating to Labeling and Instruction for Use of in vitro diagnostic} medical devices

Malaria RDTs are in vitro diagnostic medical devices (IVDs): the Latin term "in vitro" means "in glass", referring to the use of IVD outside the human body. In regulatory practice, IVDs are considered a subset of the large family of medical devices (MD), some of which make contact or are used inside the human body (from tongue depressors to pacemakers)[15]. Of note, in regulatory terms "labeling" refers to labels (for instance on the IVD packaging) as well as to instructions for use.

The International Medical Device Regulators Forum (IMDRF) (http://www.imdrf.org/) - previously the Global Harmonization Task Force (GHTF) - brings together medical device regulators from Australia, Brazil, Canada, the European Union, Japan, the United States of America, China and the Russian Federation. The World Health Organization is an official observer. IMDRF aims to accelerate international medical device (and therefore IVD) regulatory harmonization and convergence through the development of non-binding guidance documents for regulatory implementation. The guidance documents are open-access and have no restrictions on reproduction and diffusion. Relevant to this project is the guidance document "Label and Instructions for Use for Medical Devices" (GHTF/SG1/N70:2011)[15].

The International Organization for Standardization (ISO) (http://www.iso.org/iso/home.html) is a worldwide federation of national standard bodies preparing standards in liaison with international governmental and non-governmental organizations. ISO 18113 "In vitro diagnostic medical devices Information supplied by the manufacturer (labelling)" provides detailed information about labeling of IVD including terms, definitions, general requirements and labelling[18]. ISO 15223 "Medical devices-Symbols to be used with medical device labels, labelling and information to be supplied" lists symbols that convey information to the safe and effective use of the IVD [20]. ISO documents are copyrighted and should be purchased from the ISO website.

In the European Union (EU), the regulatory framework for IVDs is provided by the "In Vitro Diagnostic Medical Device Directive (IVDD 98/79/EC)"[21], operational since 2000 and currently under revision. The European Committee for Standardization (known by its French acronym CEN) further elaborated IVDD 98/79/EC: with regard to labeling, there is the European Standard EN 980 "Symbols for Use in the Labeling of Medical Devices". CEN Standards are adopted (i.e. translated in national legislation) by each of the EU member countries. Of note, ISO and CEN have close interaction: more than $30 \%$ of European Standards adopted by CEN are ISO standards and in 2013, EN980 has been replaced by the adopted EN ISO 15223 standard[20]. In addition to CEN standards, there are guidelines ("MEDDEVs") produced by experts from manufacturers and organizations involved in conformity assessment procedures. Although they are not legally binding they provide useful information; for labeling, there is the MEDDEV.2.14/3 rev.1 "IVD GUIDANCES: Supply of Instructions for Use (IFU) and other information for In-vitro Diagnostic (IVD) Medical Devices"[22].

The USA Food and Drug Administration (FDA) (http://www.fda.gov/) regulations relating to labeling of IVDs are documented in document Title 21 of the Code of Federal Regulations, Part 809 (21 CFR 809.10)[24]. Requirements are in line with those described in IMDRF guidelines and ISO/CEN standards.

A future evolution is the globally harmonized "Unique Device Identification" (UDI) of Medical Devices" - IMDRF and FDA recently published guidance documents $[17,43]$. The UDI consists of numeric and/or alphanumeric unambiguous identification and tracking of a specific medical device on the market. It includes a device as well as a production identifier and is linked to a UDI Database. The UDI carrier can be presented by readable characters and/or captured by bar codes, smart cards or other technologies. The marking of the UDI to the device or its package and to all higher levels of package is proposed to be an additional requirement for labeling.

Figure 1 Source of regulatory documents relating to labelling and instruction for use of in vitro diagnostic medical devices

labelling of box, cassette packaging, cassettes, buffer bottle and accessories. Emphasis was placed on durability (permanent printing or water-resistant labels lasting the lifespan of the RDT product), legibility (font size, letter type, see Figure 2), comprehension (use of symbols) and ease of reference (e.g. place of labelling on the box or cassette packaging, allowing quick oversight). HarT further recommended the use of the official language(s) of the country/ region of intended use (expert suggestion). In addition, it was proposed to foresee a place for affixing the Unique Device Identification (UDI, see Figure 1) label (expert suggestion). Among the information required to be displayed, a meaningful product name (revealing the intended use and antigens targeted) was recommended and (as for other RDT components), the expression of the expiry date was agreed as year and month (YYYY-MM). The information identifying the legal manufacturer should include the name, the physical address of the manufacturing site and contact details, i.e. telephone and/or fax number and website. HarT recommended also to display a clearly visible additional warning on the RDT box in case the test procedure or IFU had changed substantially. Figure $3 \mathrm{~A}$ and B display examples of the required labelling of the RDT box, including product name, product code, intended use, number of tests, information about the manufacturer as well as symbols addressing storage conditions, warnings and precautions. All relevant information needed for stock management (e.g. product identity, storage 


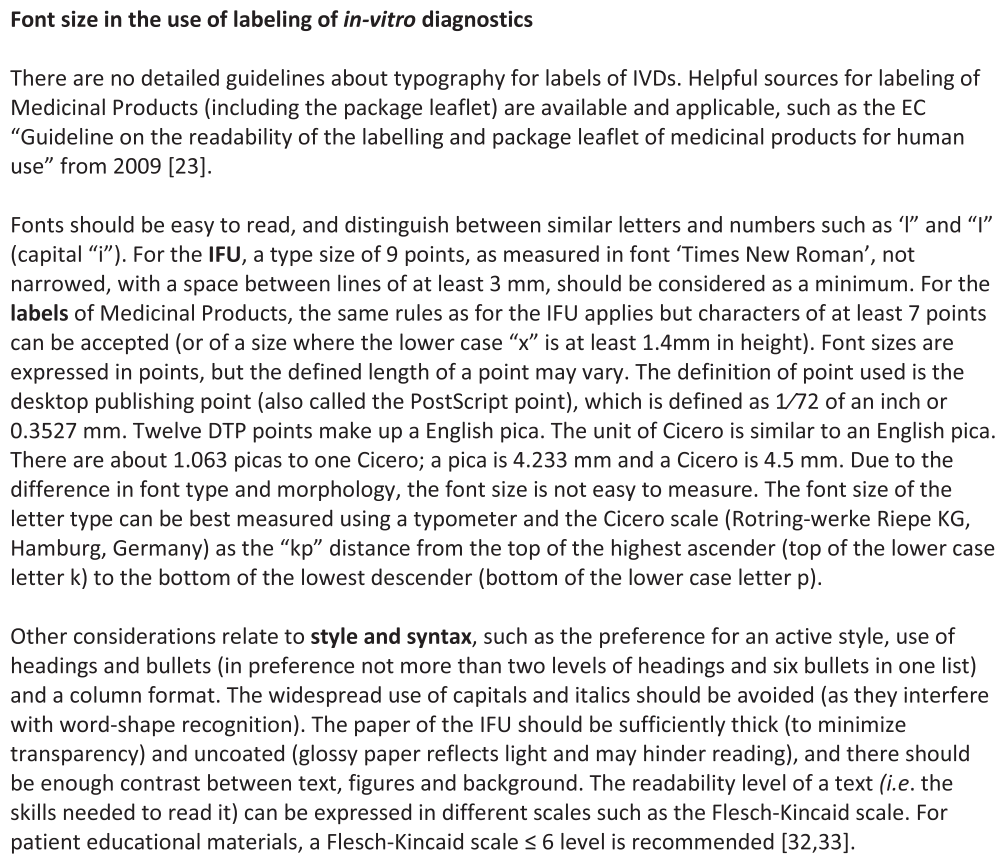

Figure 2 Font size in the use of labelling of in-vitro diagnostics.

conditions and materials provided) should also be displayed on two sides, i.e. the front side and at least one lateral side of the RDT box, except for custom/variable information such as lot number and expiry date which could be (for reasons of cost) printed on only a single side of the box (implementer suggestion).

Likewise, consensus on labelling specifications for buffer bottle, cassette packaging, desiccant packaging and accessories (specimen transfer devices, lancets, alcohol swabs and desiccants) were compiled (Additional file 2, Figure 4). Similar recommendations for labelling relating to durability, legibility and user-friendliness were made. For the cassette packaging, the consensus was to display all standard information (name, storage, warnings and precautions) on one side of the packaging and to display the custom or variable information (lot number, expiry date) on the other side; this to allow cost-effective printing and processing (implementer suggestion).

For the accessories, HarT referred to the option to display the required information on the packaging of multiple devices when it is not practicable to display it on the device itself $[15,21]$. Lancets and alcohol swabs are regulated as medical devices and are to be labeled as such; alcohol swabs are to be labeled as antiseptics (contact with tissues), not as disinfectants (contact with objects).

For labelling of the cassette, a convention for terminology and orientation of labelling was agreed (Figure 5, implementer suggestion). Prints in indelible ink are recommended over characters embossed in the cassette housing or labels glued to the reading legend (Figure 6). Orientation of the text should be parallel to the short axis, and a single unequivocal reading legend should be displayed at the right hand side of the results window. The product name should be printed on the distal side of the cassette. Specimen and buffer wells should be labeled as "1" and "2" respectively, according to the chronological order of the procedure. Plasmodium species detected should be displayed by the agreed abbreviation in the reading legend (Additional file 1 ).

A final HarT consensus document addressed the specifications for the IFU in the form of a generic template with background information and detailed instructions (Additional file 3). The instructions addressed the lay-out and style and its user-friendliness (including referral to tools for assessing readability level) as well as a referral to relevant and updated websites and studies. Recommendations were also compiled about appropriate description of IVD performance specifications. In addition an example was provided of a symbol key displaying the internationally recognized symbols relevant for IVDs, along with their explanation (expert suggestion).

\section{Discussion}

\section{Labelling assists performance, quality and safety of in vitro diagnostic medical devices (IVDs)}

Regulation of IVDs targets consistent performance and quality of products and their safety. Best international regulatory practice dictates that a manufacturer should undertake an ongoing risk assessment of the IVD 


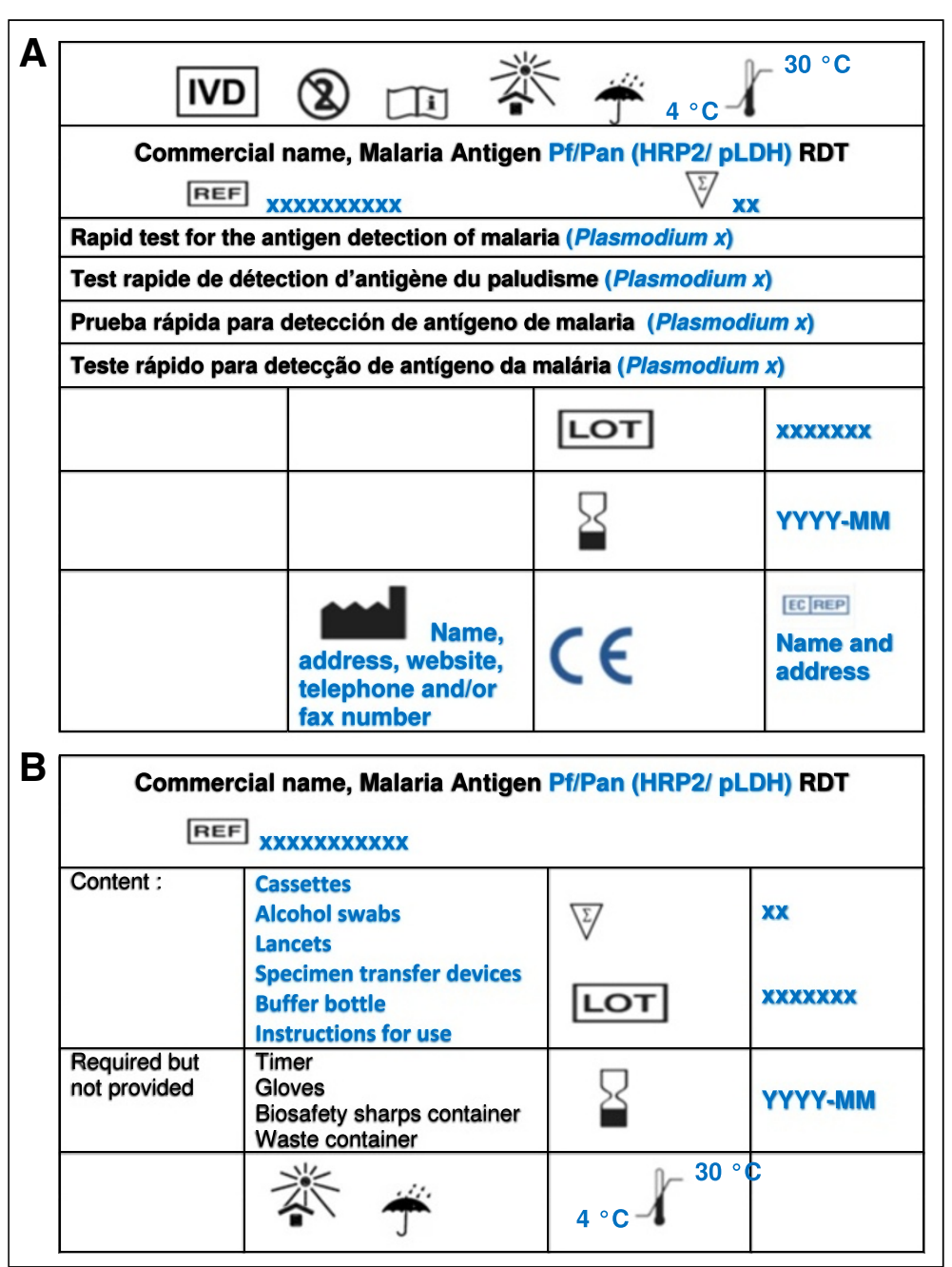

Figure 3 Examples of the required labelling of the RDT box. 3 A: top side, 3 B: lateral side. Labelling includes product name, product code, intended use, number of test, information about the manufacturer, symbols addressing storage conditions, warnings and precautions. Text in blue displays RDT product specific characteristics.

product's use and manufacture, in which all risks and their consequences (false or missed diagnosis, transmission of infections etc.) are identified and minimized by appropriate design, construction and manufacturing $[17,21]$. Communication of any residual risk to the user has to be provided by labelling, which includes labels as well as the IFU (Figure 1) $[15,17]$. As such, this communication includes warnings and precautions but also statements of known test limitations.

\section{Labelling should target the user's profile}

An explicit part of the regulatory responsibility of the manufacturer is to align communication to the level of the IVD user's education, training and expertise $[15,17]$. Medium, format, content, legibility and location of the label should be appropriate for the intended user [15]. This has particular relevance when considering the use of malaria RDTs in the private sector, the community and home-based management of malaria [4,29-31].

Guidelines for lay-out and readability, and the use of internationally recognized symbols

There are no guidelines defining labelling font sizes and styles for IVDs, but useful information can be found in pharmaceutical guidance on labelling of medicines' package leaflets referring to font, lay-out and style (Figure 2) [23]. In terms of syntax, readability is expressed by socalled reading scales such as the Flesch-Kincaid grade which expresses the U.S. grade-level equivalence of the skills required to read a particular document: for the purpose of RDTs in the present setting, a grade $\leq 6$ is 


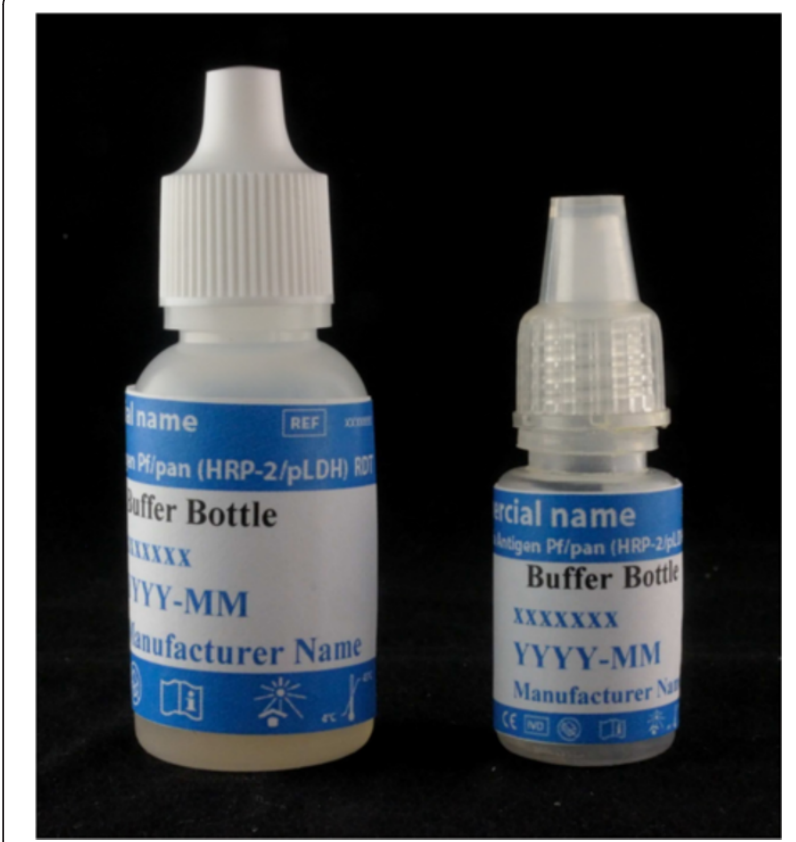

Figure 4 Labelling of the buffer bottles. Two common sizes of buffer bottles, labeled according to the HarT requirements. The label used for the large buffer bottle has a size of $4.9 \mathrm{~cm} \times 3.0 \mathrm{~cm}$; all information is displayed in a font Times New Roman, size 10 points, bold. For the small buffer bottle, a label of $4.9 \mathrm{~cm} \times 2.3 \mathrm{~cm}$ is used. Essential information (commercial name, buffer bottle, expiration, lot number) is printed in Times New Roman bold, size 9 points; other information is printed in Times New Roman bold size 7 points.

recommended, in line with the requirements for patient educational materials [32,33].

In view of swift and consistent interpretation of communication, the use of graphical symbols is promoted: symbols save space, obviate the need for translations and convey standardized and clear messages at high visual impact and noticeability $[15,20,21,34]$. Symbols described in ISO 15223 [19] may be used in CE marked IVDs without further explanation, however, comprehension of these assumed "self-explanatory" symbols may be poor particularly among untrained staff in resource limited settings [35]. Therefore, in addition to training and educational outreach, adding a symbol key (i.e. a glossary of symbols used) to the IFU is recommended, in line with FDA recommendations [36].

\section{Relevance for the RDT market}

The malaria RDT market currently comprises a plethora of products and manufacturers, with constant revisions made to products in order to improve quality and performance $[13,14]$. Although the public sector is centered around a core set of products and suppliers, this changes over time. Products in the private sector are more diverse and mostly not aligned with those products recommended by WHO and in turn, the NMCPs

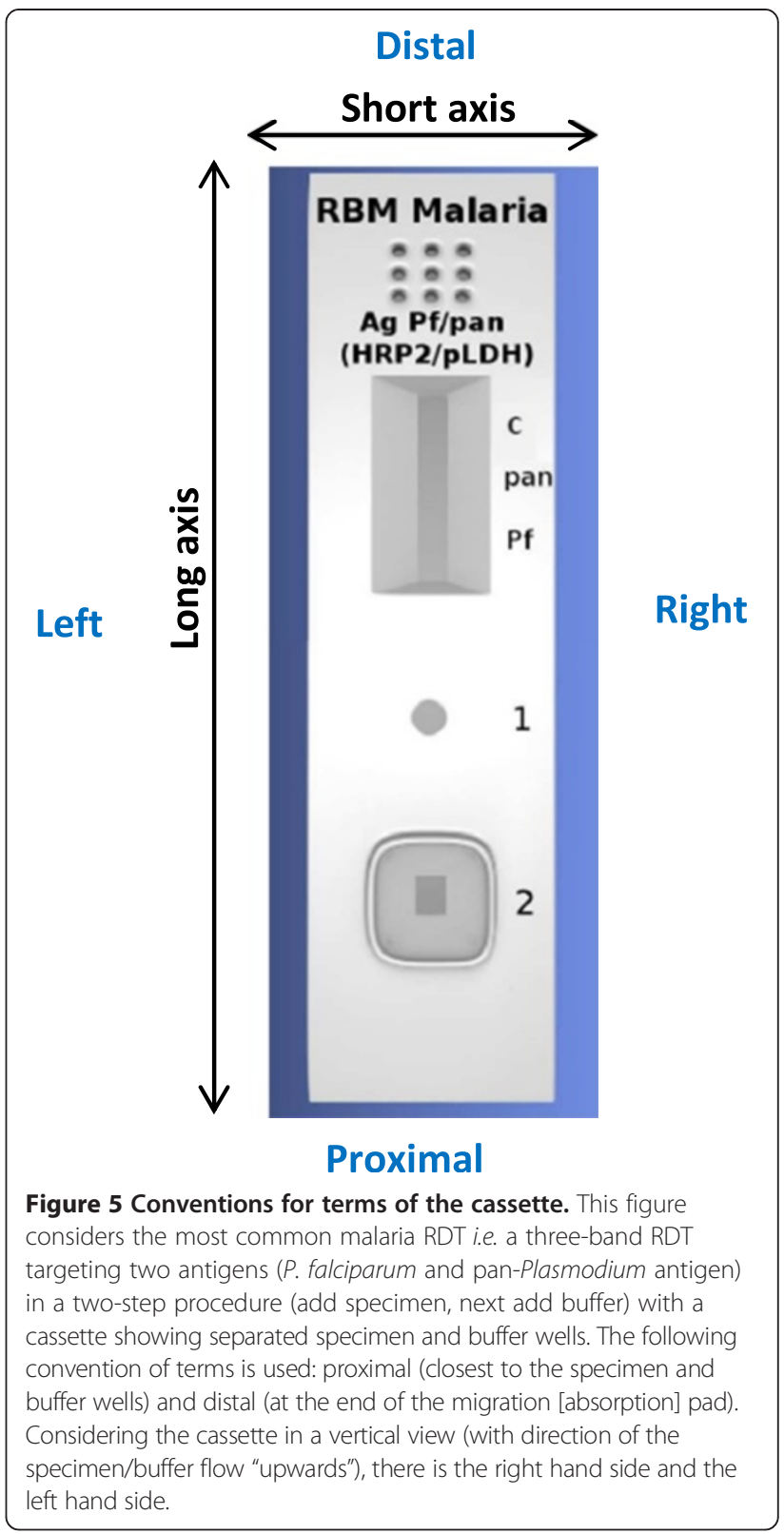

(Availability and price of malaria rapid diagnostic tests in the private health sector in 2011: results from ten nationally representative cross-sectional retail surveys. Poyer S., Goodman C et al., personal communication, [5]). Lack of quality has been noted as an important market shortcoming: the RDT market has started in an era with minimal regulatory oversight or quality standards and most resource limited settings have no effective IVD regulations or post-market surveillance in place [5]. Despite quality initiatives such as the WHO Malaria RDT Product Testing [37] and WHO-FIND Lot Testing Programmes [38] the WHO RDT Procurement Guidance [14] and the WHO Prequalification of Diagnostics and Medical Devices Programme [39], the RDT market is 



Figure 6 Malaria RDT cassettes: differences in visibility between embossed versus printed characters. The figures demonstrate the difference between visibility/readibility of embossed versus printed characters. A shows the cassettes in tangential ("side") light: the embossed characters are well discernable. B shows the cassettes in regular (direct) light: the embossed characters are less discernable - visibility will even decrease in low light conditions.

mainly driven by the economics of scale [5]. Prices and benefit margins have declined over recent years and high orders, short lead times and an unpredictable market may compromise product quality [5].

Another perceived difficulty of RDT use was confirmed by interviews with implementers from the public sector in the present study but also recorded from the private sector [40]: weaknesses referred to aspects of labelling and IFU, as well as RDT accessories (lancets, alcohol swabs and specimen transfer devices). Differences in these aspects contribute to the difficulties encountered in changing from one RDT product to another, a practice dictated by the open competitive tenders in the RDT market [5].

The present study assessed the available evidence to identify best practices in labelling and IFUs tailored to malaria RDTs and associated accessories. In the initial phases of the project, identifying a representative group of implementers involved in daily use of RDTs was challenging; however, the consultation on the original working document [26] and subsequent iterative review processes gradually included a large number of participants whose activities and roles are highly related to malaria RDTs use in the field, as well as their manufacturing, their procurement and use, and their regulation. Should this set of recommendations, most of which are derived from existing international guidelines, be adopted broadly, it is expected to make RDTs more user friendly and to facilitate product interchangeability. Moreover they may contribute to increased quality and improved performance of RDTs. Based on the information of participating manufacturers, most - if not all - recommendations can be achieved at a reasonable cost and in a short time span. In addition to producing a meaningful list of recommendations and reference documents, this study has also stimulated the dialogue between users, implementers, buyers, regulatory experts and manufacturers. The 
consensus document generated through this collective process steered by RBM [28], may also be of value to rapid diagnostic tests and IVDs addressing other infectious diseases which is relevant since most manufacturers have RDTs in their portfolio specific for multiple diseases.

\section{The way forward, benefits of harmonization/labelling}

The present HarT recommendations offer an opportunity for improving consistency and harmonization of RDT product characteristics and provide a comprehensive resource upon which international recommendations and tender specifications can be based. Various channels may be exploited for diffusion and application to ensure maximum uptake. Furthermore, the recommendations may also guide and orient emerging national IVD regulations and regional initiatives such as the Pan-African Harmonization Working Party on Medical Devices and Diagnostics (PAHWP) [41]. Synergistic integration of the recommendations into assessment procedures and procurement practices - particularly when extended to other IVDs - will add quality as a driving market factor, increase awareness about quality standards for IVDs among end-users and provide incentives to manufacturers to further invest in robust quality systems.

\section{Conclusions}

The present document together with its additional files compiled proposes best practices in labelling and IFU for malaria RDTs. It is expected that compliance with these best practices will increase harmonization among the different malaria RDT products available on the market and improve their user-friendliness.

\section{Additional files}

\section{Additional file 1: Suggested terms and abbreviations related to} malaria RDTs.

Additional file 2: Requirements for the labelling of malaria RDT kit components: box, cassette packaging, cassette, buffer bottle and accessories.

Additional file 3: Generic template for Instructions for Use (IFU).

\section{Abbreviations}

CLSI: Clinical and Laboratory Standards Institute; EC: the European Commission; FDA: Food and Drug Administration; GHTF: Global Health Task Force; HarT: Harmonization Task force; IFU: instructions for use; IMDRF: International Medical Device Regulators Forum; ISO: International Organization of Standardization; ITM: Institute of Tropical Medicine; IVD: in vitro diagnostics; mRDT: Malaria rapid diagnostic test; NMCP: National Malaria Control Programme; P: Plasmodium; PAHWP: Pan-African Harmonization Working Party on Medical Devices and Diagnostics; RBM: Roll Back Malaria Partnership; RDT: Rapid diagnostic test; UDI: Unique device identification; WHO: World Health Organization.

\section{Competing interests}

The authors declare that they have no competing interests.

\section{Authors' contributions}

JVE, MA, JC, TV conceived the study idea on behalf of Roll Back Malaria Partnership (RBM); JJ, BB, PG and ESC participated in the study design and $J V E, M A, J C, T V$ and $J J$ in the overview and coordination. BB, PG, ESC and JJ compiled the international standards, regulatory documents and published literature, BB and PG designed and performed the questionnaires. JJ, PG, ESC and BB drafted the initial report in preparation of the RBM Stakeholder consultation meeting. All authors co-organized the RBM Stakeholder consultation meeting and actively participated to the subsequent task force and the refinement and finalization of the recommendations. JJ drafted the initial version of manuscript, all authors contributed to revisions and amendments and have approved the final version of the manuscript.

\section{Acknowledgments}

We thank the Roll Back Malaria mRDT Harmonization Taskforce (HarT) members for their contribution in the drafting and approval of the consensus documents. The WHO Prequalification of In Vitro Diagnostics Programme contributed to the preparation of this manuscript.

\section{Funding}

This study was funded by Roll Back Malaria Partnership and by the Directorate General for Development Cooperation of the Belgian Government through the Network Program on Laboratory Quality Management, Project 3.21. The funders had no role in the study design, data collection and analysis or the preparation of the manuscript.

\section{Author details}

'Department of Clinical Sciences, Institute of Tropical Medicine, Antwerp, Belgium. ${ }^{2}$ Department of Microbiology and Immunology, University of Leuven, Leuven, KU, Belgium. ${ }^{3}$ Malaria Branch, Division of Parasitic Diseases and Malaria, Center for Global Health, Centers for Disease Control and Prevention, Atlanta, GA, USA. ${ }^{4}$ Roll Back Malaria Partnership, Geneva, Switzerland. ${ }^{5}$ The Global Fund to Fight AIDS, Tuberculosis and Malaria, Geneva, Switzerland. ${ }^{6}$ The Foundation for Innovative New Diagnostics, Geneva, Switzerland. 'WHO Global Malaria Programme, World Health Organization, Geneva, Switzerland. ${ }^{8}$ Clinton Health Access Initiative, Boston, MA, USA.

Received: 17 October 2014 Accepted: 9 December 2014 Published: 17 December 2014

\section{References}

1. World Health Organization: World Malaria Report. 2013 [http://www.who.int/ iris/bitstream/10665/97008/1/9789241564694_eng.pdf?ua=1]

2. World Health Organization: Guidelines for the treatment of malaria, second edition. 2010 [http://whqlibdoc.who.int/publications/2010/ 9789241547925_eng.pdf]

3. World Health Organization: T3: Test. Treat. Track. Scaling up diagnostic testing, treatment and surveillance for malaria; 2012 [http://www.who.int/entity/ malaria/publications/atoz/test_treat_track_brochure.pdf?ua=1]

4. Counihan H, Harvey SA, Sekeseke-Chinyama M, Hamainza B, Banda R, Malambo T, Masaninga F, Bell D: Community health workers use malaria rapid diagnostic tests (RDTs) safely and accurately: results of a longitudinal study in Zambia. Am J Trop Med Hyg 2012, 87:57-63.

5. UNITAID: Malaria Diagnostics Market Landscape; 2012 [http://www.unitaid. eu/images/marketdynamics/publications/UNITAID\%20Malaria\% 20Diagnostics\%20Market\%20Landscape_2012.pdf]

6. Batwala V, Magnussen P, Nuwaha F: Are rapid diagnostic tests more accurate in diagnosis of Plasmodium falciparum malaria compared to microscopy at rural health centres? Malar J 2010, 9:349.

7. Batwala V, Magnussen P, Hansen KS, Nuwaha F: Cost-effectiveness of malaria microscopy and rapid diagnostic tests versus presumptive diagnosis: implications for malaria control in Uganda. Malar J 2011, 10:372.

8. de Oliveira AM, Skarbinski J, Ouma PO, Kariuki S, Barnwell JW, Otieno K, Onyona P, Causer LM, Laserson KF, Akhwale WS, Slutsker L, Hamel M: Performance of malaria rapid diagnostic tests as part of routine malaria case management in Kenya. Am J Trop Med Hyg 2009, 80:470-474.

9. Maltha J, Gillet P, Jacobs J: Malaria rapid diagnostic tests in travel medicine. Clin Microbiol Infect 2013, 19:408-415. 
10. UNITAID: Malaria Diagnostics Market Landscape Update; 2013 [http://www. unitaid.eu/images/projects/malaria/UNITAID_2013_Update_Malaria_ Diagnostics_Market_Landscape.pdf]

11. World Health Organization: Universal access to malaria diagnostic testing. An operational manual; 2011 [http://whqlibdoc.who.int/publications/2011/ 9789241502092_eng.pdf]

12. Maltha J, Gillet $\bar{P}$, Jacobs J: Malaria rapid diagnostic tests in endemic settings. Clin Microbiol Infect 2013, 19:399-407.

13. UNITAID: Malaria Diagnostic Technology Landscape; 2011 [http://www. unitaid.eu/marketdynamics/malaria-diagnostics-landscape]

14. World Health Organization: Good practices for selecting and procuring rapid diagnostic tests for malaria; 2011 [http://whqlibdoc.who.int/publications/ 2011/9789241501125_eng.pdf]

15. The Global Harmonization Task Force: GHTF/SG1/N70:2011 Label and Instructions for Use for Medical Devices; 2011 [http://www.imdrf.org/docs/ ghtf/final/sg1/technical-docs/ghtf-sg1-n70-2011-label-instruction-usemedical-devices-110916.pdf]

16. International Medical Device Regulators Forum: IMDRF/WG/N7FINAL:2013 UDI guidance. Unique Device Identification (UDI) of Medical Devices; 2013 [http://www.imdrf.org/docs/imdrf/final/technical/imdrf-tech-131209-udiguidance.pdf]

17. The Global Harmonization Task Force: GHTF/SG1/N68:2012 Essential Principles of Safety and Performance of Medical Devices; 2012 [http://www.imdrf.org/ docs/imdrf/final/technical/imdrf-tech-131209-udi-guidance-140901.pdf]

18. ISO 15189-2007: Medical laboratories. Particular requirements for quality and competence; 2007 [http://www.iso.org/iso/home.html]

19. ISO 15223-1-2012: Medical devices - Symbols to be used with medical device labels, labelling and information to be supplied; 2012 [http://www.iso.org/iso/ home.html]

20. ISO 18113-2009: In vitro diagnostic medical devices - Information supplied by the manufacturer (labelling) - Part 1-5; 2011 [http://www.iso.org/iso/home.html]

21. European Commission: Directive 98/79/EC of the European parliament and of the council of 27 October 1998 on in vitro diagnostic medical devices; 2009 [http://eur-lex.europa.eu/LexUriServ/LexUriServ.do?uri=CONSLEG:1998L0079: 20090807:EN:PDF]

22. European Commission: MEDDEV. 2.14/3 rev.1. Guidelines on medical devices. IVD guidances: Supply of Instructions For Use (IFU) and other information for In-vitro Diagnostic (IVD) Medical Devices. A guide for manufacturers and notified bodies; 2007 [http://ec.europa.eu/health/medical-devices/files/meddev/ 2_14_3_rev1_ifu_final_en.pdf]

23. European Commission: ENTR/F/2/SF/jr (2009)D/869 Guideline on the readability of the labelling and package leaflet of medicinal products for human use. Revision 1; 2009 [http://ec.europa.eu/health/files/eudralex/vol-2/ c/2009_01_12_readability_guideline_final_en.pdf]

24. U.S.Food and Drug Administration: 21 CFR 809.10 Title 21 of the Code of Federal Regulations, Part 809.10. Labeling for in vitro diagnostic products; 2011 [http://www.gpo.gov/fdsys/granule/CFR-2011-title21-vol8/CFR-2011-title21vol8-sec809-10/content-detail.html]

25. Clinical and Laboratory Standards Institute (CLSI): Procedures and devices for the collection of diagnostic capillary blood specimens; Approved standard Sixth edition. Wayne, PA: Clinical and Laboratory Standards Institute; 2008. GP42-A6, Vol. 28, No. 25.

26. Procurement and Supply Chain Management Working Group - Diagnostics Work Stream: Enhanced Malaria RDT Harmonization. RDT Consultation - Working Document, version III.2, November 15th 2013 [http://www.rollbackmalaria.org/ partnership/wg/wg_procurementsupply/docs/2013-rdt-consultation/RDT Consultation_Annex6.pdf]

27. Roll Back Malaria Partnership: The Malaria RDT landscape Workshop: Update and perspectives on RDT prequalification. Geneva. 23/05/2011.

28. Roll Back Malaria Partnership and Institue of Tropical Medicine: Report of the Stakeholder Consultation on enhanced Harmonization of malaria Rapid Diagnostic Tests, 3-5 December. Belgium: ITM Antwerp; 2013 [http://www. rollbackmalaria.org/partnership/wg/wg_procurementsupply/docs/2013-rdtconsultation/RDTconsultation_report.pdf]

29. Kamal-Yanni MM, Potet J, Saunders PM: Scaling-up malaria treatment: a review of the performance of different providers. Malar J 2012, 11:414.

30. Special Programme for Research and Training in Tropical Diseases (TDR): Malaria RDTs in private sector; 2014 [http://www.wpro.who.int/malaria/sites/ rdt/using_rdts/rdt_private_sector.html]

31. World Health Organization: World Malaria Report; 2012 [http://www.who.int/ iris/bitstream/10665/78945/1/9789241564533_eng.pdf?ua=1]
32. Gillet P, Maltha J, Hermans V, Ravinetto R, Bruggeman C, Jacobs J: Malaria rapid diagnostic kits: quality of packaging, design and labelling of boxes and components and readability and accuracy of information inserts. Malar J 2011, 10:39.

33. Wallace LS, Keenum AJ, Roskos SE, Koopman RJ, Young KG: Blood glucose monitor quick reference guides: are they suitable for patients? Diabetes Technol Ther 2008, 10:11-15.

34. Liu L, Hoelscher U, Gruchmann T: Symbol comprehension in different countries: experience gained from medical device area. Workshops Proceedings der 5 Fachübergreifenden Konferenz Mensch und Computer.Wien: Oesterreich 2005, 81:7.

35. Hermans V, Monzote L, Van den Sande B, Mukadi P, Sopheak T, Gillet P, Jacobs J: Assessment of the knowledge of graphical symbols labelled on malaria rapid diagnostic tests in four international settings. Malar J 2011, 10:331.

36. U.S.Food and Drug Administration: Guidance for Industry and FDA staff. Use of Symbols on Lables and in Labeling of In Vitro Diagnostic Devices Intended for Professional Use; 2004 [http://www.fda.gov/downloads/MedicalDevices/ DeviceRegulationandGuidance/GuidanceDocuments/ucm085679.pdf]

37. World Health Organization: WHO Malaria Rapid Diagnostic Test Product Testing Programme; [http://www.who.int/malaria/news/2014/ rdt_call_for_testing_round6/en/]

38. Foundation for Innovative New Diagnostics (FIND): WHO-FIND Malaria RDT Lot Testing Programme. [http://www.finddiagnostics.org/programs/malariaafs/malaria/rdt_quality_control/lot_testing/]

39. World Health Organization: WHO Prequalification of In Vitro Diagnostics Programme. [http://www.who.int/diagnostics_laboratory/evaluations/en/]

40. Streat E: RDTs in the private sector - PSM challenges. 10th meeting of the Procurement and Supply Management Working Group (PSM-10); [http://www. rollbackmalaria.org/partnership/wg/wg_procurementsupply/docs/ 10psmwgGroupB_Streat.pdf]

41. Pan African Harmonization Working Party on Medical Devices and Diagnostics; [http://www.pahwp.org/]

doi:10.1186/1475-2875-13-505

Cite this article as: Jacobs et al:: Harmonization of malaria rapid diagnostic tests: best practices in labelling including instructions for use. Malaria Journal 2014 13:505.

\section{Submit your next manuscript to BioMed Central and take full advantage of:}

- Convenient online submission

- Thorough peer review

- No space constraints or color figure charges

- Immediate publication on acceptance

- Inclusion in PubMed, CAS, Scopus and Google Scholar

- Research which is freely available for redistribution 